\title{
FORECASTING ALGAL BLOOM LAGS AND STABILITY IN A WATERSHED*
}

\author{
MACKENZIE JONES ${ }^{\dagger}$, ALISON FRIDERES ${ }^{\ddagger}$, BAILEY KRAMER ${ }^{\S}$, AND FACULTY \\ ADVISOR: KEITH WOJCIECHOWSKI $\$$
}

\begin{abstract}
Near Menomonie, Wisconsin the lakes suffer from algae blooms during the warm summer months. Mathematical models describing the cyanobacteria population dynamics are studied with the intent of analyzing the conditions under which the populations grow and stabilize. Two models are considered, one for forecasting the population as the lake turns toxic from excess biomass after a flushing event occurs, and the other for estabilishing an algal bloom stability condition. These models are proposed for consideration to test the effectiveness of solutions put forth to ameliorate the algal bloom problem.
\end{abstract}

Key words. logistic growth, convection-diffusion, algal-bloom

1. Introduction. Eutrophication refers to the process of an ecosystem becoming more productive due to nutrient enrichment that stimulates primary producers [2]. This process has led to the deterioration of lakes worldwide [5]. While eutrophication is thought to be part of the natural ontogeny of some lakes, it can be sped up through human activities [2]. Human driven eutrophication is a process that occurs rapidly and it can be difficult to reverse. It is caused mainly by point and non-point source inputs of nitrogen and phosphorus. These sources can be traced largely to agricultural practices, deforestation, and the release of sewage into the watershed. The resulting change in water chemistry leads to a shift in species composition where phytoplankton dominate macrophytes [2]. These changes often lead to decreased water quality and increased algal blooms.

Algal blooms cause unwanted outcomes in impacted watersheds. Thick blooms around the edges of a lake can prevent people from swimming and taking watercraft out onto the lake. A degraded lake causes surrounding houses to be priced lower and area tourism to decrease $[1,8]$. Additionally, these blooms can produce liver toxins that are potentially deadly to wildlife utilizing the lake, including humans [9]. One of the main producers of toxic algal blooms is the cyanobacteria Microcystis aeruginosa, a bacterium that lives in eutrophic freshwater. M. aeruginosa are not greatly impacted by competition or predators. Cyanobacteria are able to outcompete other species for light by employing chlorophylls, carotenoids, and phycobilins that allow them to obtain light energy from areas of the spectrum that other taxa cannot utilize [10]. The optimal growth temperature of cyanobacteria is much higher than other eukaryotic taxa and cyanobacterial blooms have been associated with an increase in local water temperatures. This means that cyanobacteria are able to bring their surrounding environment to a temperature that is both better for their own optimal growth and detrimental to the growth of their competitors. Furthermore, M. aeruginosa are able to utilize gas vesicles which can be manipulated to change size and density in order to regulate buoyancy. These gas vesicles, along with a resistance to ultraviolet radiation, allow the cyanobacteria to shade out non-buoyant phytoplankton and macrophytes

*Submitted to the editors DATE.

Funding: This work was funded by the National Science Foundation SMA grant no. 135738.

$\dagger$ Department of Mathematics, University of Akron, Akron, OH (mlj54@zips.uakron.edu).

${ }_{\ddagger}^{\ddagger}$ Department of Mathematics, Simpson College, Indianola, IA (allison.frideres@my.simpson.edu).

$\S$ Department of Mathematics, Statistics, and Computer Science, University of Wisconsin Stout, Menomonie, WI (kramerb0587@my.uwstout.edu, wojciechowskik@uwstout.edu). 
[10].

There is evidence that $M$. aeruginosa are able to use multiple different methods to avoid predation. Many strains can produce cyanotoxins, which are broad spectrum and make the cyanobacteria difficult to consume. M. aeruginosa are also covered in a gelatinous coating. There is evidence that this makes them indigestible to many species and there is further evidence that some strains are still viable after passing through the gut of grazers. Moreover, the cyanobacteria accumulate in overwhelming numbers. At such high densities it is possible that the negative impact any grazers may have on the cyanobacteria population is outweighed by the size of that population. Furthermore, while there exist bacteria viruses in the lake that cause cyanobacteria death, the numbers are not sufficient to greatly affect the large cyanobacteria population [9]. The large size and density of the cyanobacteria population also reduces the ability of grazers to reach and consume other prey which ultimately decreases the fitness of the grazer.

These adaptations decrease pressures from predation and competition causing cyanobacteria to be more limited by environmental factors such as a need for sunlight, an optimum growth temperature, a high nitrogen to phosphorus ratio, low turbulence, and a long residence time. Cyanobacteria may favor strong sun exposure because they excel in waters that periodically exceed 20 degrees Celsius, with surface forming genera reaching optimal temperatures around 25 degrees Celsius. $M$. aeruginosa is a non- $\mathrm{N}_{2}$ fixing species, meaning it cannot transform inorganic forms of nitrogen into a viable form for photosynthesis [10]. In order for cyanobacteria to grow and produce organic matter there must be a large amount of nitrogen freely available to them.

Low turbidity, long residence times, and calm surface waters are also needed for optimal growth [10]. In order to uptake nutrients, the cyanobacteria utilize their gas vesicles to sink to the bottom of the lake and then float back up to the surface to get necessary sunlight. If a lake is highly turbulent, it becomes more difficult for $M$. aeruginosa to maintain an optimal vertical position, allowing non-motile species to have a greater competitive advantage. Cyanobacteria are able to consume more phosphorus than necessary for biological production and store it for later use, as well as sequester essential trace nutrients like iron $[7,10]$. Long residence times, without flushing the lake, allow cyanobacteria to uptake high amounts of phosphorus and produce blooms before a high turbulence event occurs. High flow events followed by periods of long residence time can be beneficial to cyanobacteria as the inflow often brings more nutrients into the lake [10]. If growth conditions are met, it takes approximately 5-7 days for the cyanobacteria to double in population [9].

These ideal growth conditions occur seasonally in Tainter Lake, a 1605 acre eutrophic reservoir in Dunn County, Wisconsin [18]. The Red Cedar and Hay Rivers inflow into the lake which was created by damming the Red Cedar River downstream. Tainter Lake outflows into the Red Cedar River toward Lake Menomin, a lake with a similar bloom situation. In general, blooms tend to be more severe in late summer, when there is often less rainfall and warmer temperatures. In Tainter Lake the phosphorus levels are deemed eutrophic based on the trophic status index. These high levels are caused from phosphorus in the sediment, agricultural runoff, and inputs from several other eutrophic lakes in the watershed [16].

The Tainter Lake reservoir has a maximum depth of approximately 11.3 meters and an average depth of about 4.3 meters [18]. Unlike many previously studied lakes with similar bloom conditions, Tainter Lake has a general lack of stratification with regards to water temperature, since stratification primarily occurs in deeper lakes when there is seasonal turnover. Figure 1 shows temperature stratification based 


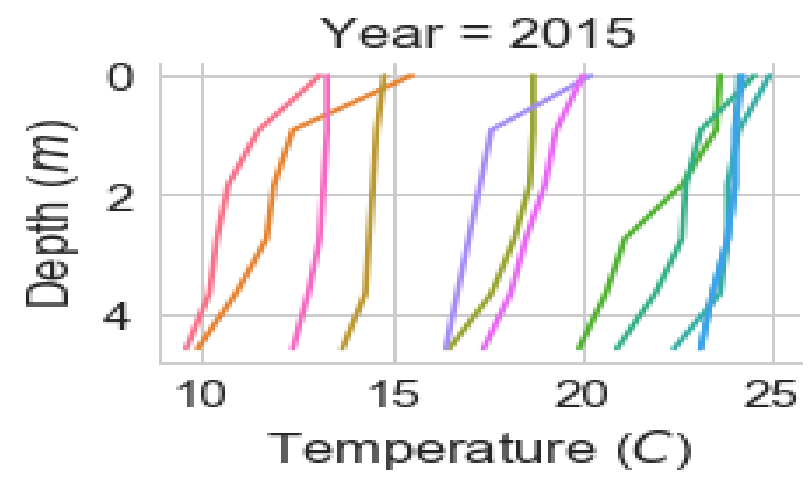

FIG. 1. Temperature stratification based on depth of Tainter Lake with different colors representing data collected on different days

on depth of Tainter Lake in the year 2015. The different colors represent different days the temperature measurements were taken, from April to October. Warmest temperatures occur from June to August and are represented by the green and blue lines. The coldest temperatures occur in April to May and September to October and are represented by the pink and orange lines. All lines are mostly vertical meaning there is no thermocline or temperature differences at different depths of the lake.

A search of the literature reveals that existing models have not been calibrated to conditions similar to those found in Tainter Lake. Therefore a modified model is needed in order to test proposed mitigation measures to cyanobacterial dominance in lakes with these conditions. The modified model could then be run with the proposed parameters to see if there would be any decrease in the cyanobacteria population.

Two models are proposed. The first is an initial value problem (IVP) containing an ordinary differential equation. This IVP is a modified logistic model for forecasting the chlorophyll-a concentration after flushing event has occurred and the lake has resumed calm surface waters to precipitate optimal growth. The advantage of this model is that it is simple to understand the simulation results and more simulations can be run with this model. The disadvantage of this model is that it can only predict chlorophyll concentration over shorter time spans of one month.

This model was motivated to understand the short-term growth patterns of algae. After a rainstorm, most algae are flushed out of the lake and phosphorus runoff is channeled into the lake. The low population of algae takes approximately 5 days to double its population and several weeks to reach a saturation concentration. Since there is a lag time for the algae to uptake the phosphorus nutrients and grow, it does not automatically seem apparent that excess phosphorus leads to algae growth. This model was created to dispel the misunderstanding that phosphorus does not cause algae growth.

The second is an initial boundary value problem (IBVP) containing a partial differential equation. This IBVP is a convection diffusion equation with a source term used to construct a bloom stability condition solely dependent upon the sinking velocity of the bacteria and the euphotic layer. If lake conditions cause this condition to fall below a certain threshold, then the $M$. aeruginosa population will collapse. The advantage to this model is that it helps us understand the biologic and limnologic conditions that lead to algae blooms. The disadvantage is that it is more difficult to 
understand and run numerical experiments with.

This model was motivated to understand the longer-term growth patterns of algae. Instead of examining chlorophyll-a concentration over time, this model is used for predicting whether the lake conditions are conducive for an algae bloom. This model gives us solutions to bring about population collapse by varying the turbulence of the water or by manipulating the algae growth rate.

This paper is organized as follows. Section 2.2 contains a description of Tainter Lake and examines the IVP used to forecast the chlorophyll-a concentration. The IVP is solved analytically as well as numerically to generate an envelope of solutions dependent upon a variety of input parameters. Section 3 analyzes the IBVP used to model the chlorophyll-a concentration as function of time and depth. The IBVP is nondimensionalized and analytically solved to arrive at a threshold for the sinking velocity and euphotic layer.

This paper contributes to the literature by modifying current models to fit the unique limnogic conditions of Tainter Lake, being a shallower lake with no thermocline. In addition, we provide two models with different ways of thinking about the problem from a short term and longer term persepective. Two models were needed because the first provides understanding of how algae uptake phosphorus to grow and take several weeks to reach a bloom level. The next model was necessary to discover the biologic and limnogic conditions that will bring about a population collapse. Both models can be used as public policy tools to understand the various options to solve the algal bloom problem.

\section{Chlorophyll Forecast.}

2.1. Lake Conditions. The model used to forecast the chlorophyll-a concentration is primarily informed by field observations obtained by James et. al. through a research experience for undergraduates (REU) and the Wisconsin Department of Natural Resources (DNR) [4, 17]. Weather data, such as rainfall, was collected by Weather Underground at Colfax, WI, about 8 kilometers from Tainter Lake [15]. Daily flow data was retrieved from the United States Geographical Survey (USGS) [14].

The summer of 2015 was not considered a drought year, receiving regular rainfall that allowed for minor to major flushing. Thus, the blooms experienced were generally not as severe as in previous years. For the scope of this paper, a chlorophyll-a measurement greater than or equal to $40 \mu \mathrm{g} / \mathrm{L}$ is considered a bloom event. The weather and flow conditions, as well as the occurrence of bloom conditions in the chlorophyll data, are shown in Figure 2. Here, the purple squares represent bloom events and the green circles represent non-bloom events. Blooms generally occurred in periods of warmer weather and lower flow. Figures 1 and 2 are used to illustrate the conditions of Tainter Lake. While these data are not used in vefirying either model proposed they are used in justifying why new models are proposed. The conditions of Tainter Lake differ from other lakes studied so new models and calibrations were needed.

One significant flushing event occurred in early July of 2015, as seen in Figure 3. Bloom events were not recorded for several weeks following the flushing event. A major bloom event, the highest in the recorded data at a CHL-a level of $197.38 \mu \mathrm{g} / \mathrm{L}$, was recorded approximately three weeks after the flushing occurred. This lag indicates that a spike in rainfall flushes the chlorophyll-a from the lake and the M. aeruginosa subsequently recovers. The population consumes the latent and newly introduced resources to grow over this period before it reaches an equilibrium. A modified logistic model can capture this behavior and is used to forecast the chlorophyll-a concentra- 


\section{FORECASTING ALGAL BLOOM LAGS AND STABILITY}
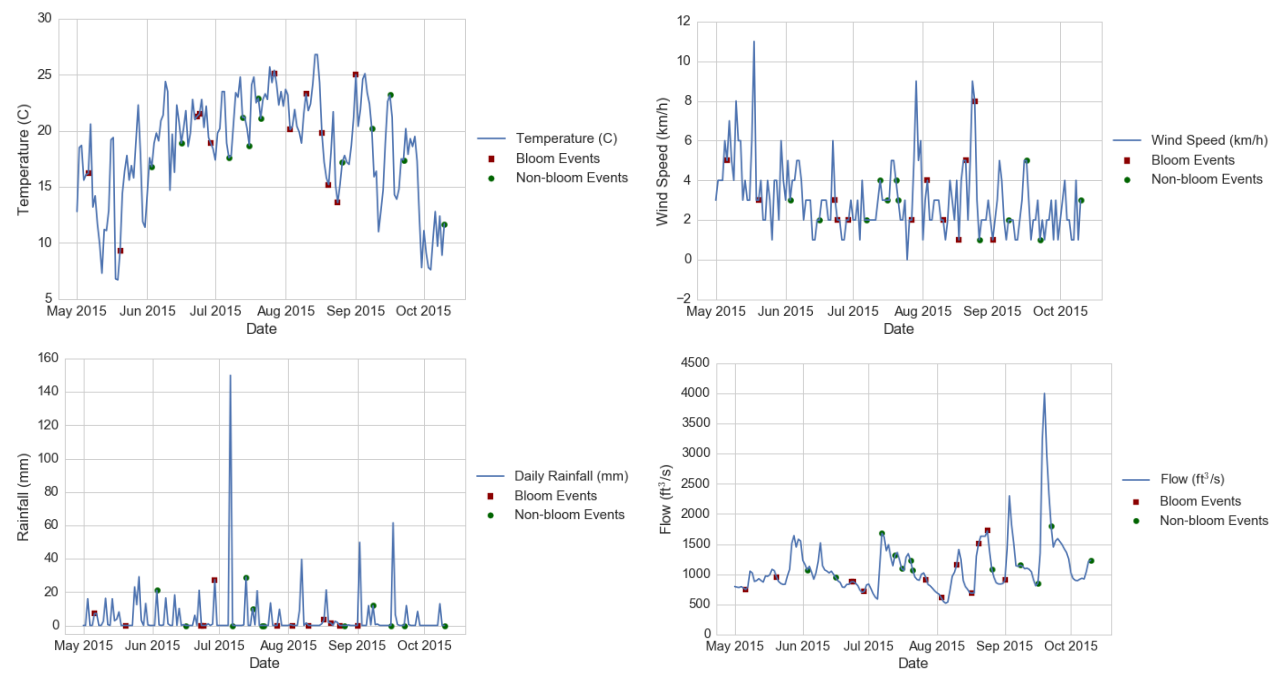

Fig. 2. May 1 - October 10, 2015: Daily temperature (C), wind speed $(\mathrm{km} / \mathrm{h})$, rainfall $(\mathrm{mm})$, and flow readings $\left(f t^{2} / s\right)$

tion. This data is used to verify model (2.1).

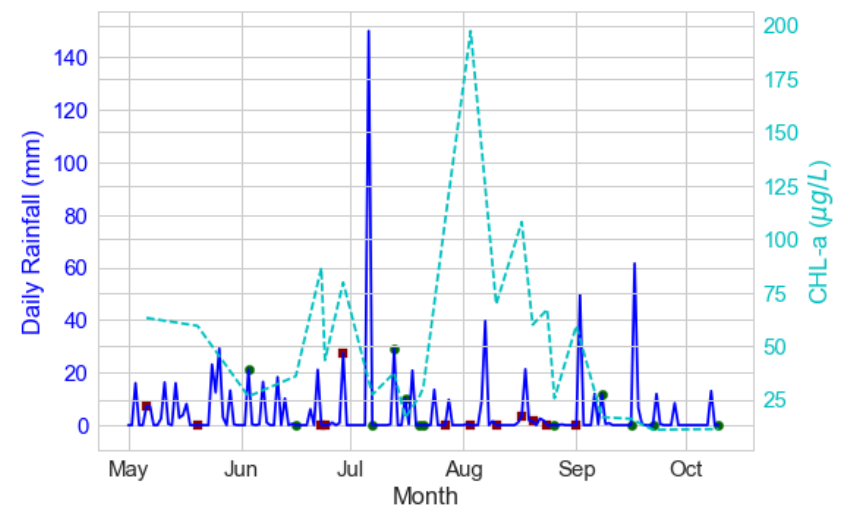

FIG. 3. May 1 - October 10, 2015: Daily rainfall $(\mathrm{mm})$ and $C H L-a(\mu \mathrm{g} / \mathrm{L})$ trends in Tainter Lake.

There is no standard chlorophyll-a measurement that denotes a bloom, the only requirement is a rapid increase in algal population. Since $40 \mu \mathrm{g} / \mathrm{L}$ is enough to cause discoloration of the water and classifies the lake as eutrophic, this estimate is used for a bloom level event. The result that a bloom occurs 3 weeks after a flushing event is robust to changes in this definition of an algae bloom. Our results are consistent for any threshold level chosen between 30 to $110 \mu \mathrm{g} / \mathrm{L}$. If a level under $30 \mu \mathrm{g} / \mathrm{L}$ is chosen as the threshold value then it only takes 1 week for the algae population to reach bloom levels after a flushing event. If a level over $110 \mu \mathrm{g} / \mathrm{L}$ is chosen as the threshold value then it takes 4 weeks for the algae population to reach bloom levels. Regardless of the threshold value chosen, the results display the behavior that there is a time lag between the rainfall event and an algal bloom. In addition, as will be 
shown in Section 2.2, higher growth rates will cause the algae population to reach the steady state more quickly.

2.2. Forecast Model. A logistic growth model was adapted from [13] in order to simply and accurately forecast chlorophyll levels. The equation provided in [13] predicts chlorophyll concentration under nutrient-limited circumstances in an impoundment over a period of a few days. However, the exponential model is realistic for short-term growth and since it was desirable to forecast chlorophyll levels over a monthly period a carrying capacity term was added to place a bound on the concentration. A longer period was required to predict concentration levels when a flushing event might not occur within 21 days. Such periods might be the result of a drought or simply low rainfall levels during the summer. During such periods the lake becomes noisome and residents report conditions surrounding the lake are intolerable. These so-called "saturation periods" may be cause of low property values for homes surrounding the lake [11].

The proposed logistic model, written as an initial value problem (IVP) is,

$$
\left\{\begin{array}{l}
\frac{d C}{d t}=C_{i} F+(\mu-F)\left(1-\frac{C}{K}\right) C \\
C(0)=C_{0}
\end{array}\right.
$$

where $C$ is the chlorophyll concentration at any point of the lake, $K$ is a saturation parameter, $C_{i}$ is the concentration of chlorophyll at the inflow of the lake, $F$ is the flushing rate, and $C_{0}$ is the initial concentration.

\begin{tabular}{cllll}
\hline Parameter & Description & Value & Units & Source \\
\hline Chlorophyll & Forecast & & & \\
$C(0)$ & Initial chlorophyll & 10 & $\mu \mathrm{gL}^{-1}$ & James [4] \\
$C_{i}$ & Inflow chlorophyll & 10 & $\mu \mathrm{gL}^{-1}$ & James [4] \\
$\mu$ & Chlorophyll growth rate & 0.30 & $d^{-1}$ & Søballe [13] \\
$F$ & Flushing rate & 0.13 & $d^{-1}$ & DNR [16] \\
$C_{\infty}$ & Steady state chlorophyll & $175 \pm 29$ & $\mu \mathrm{gL}^{-1}$ & DNR [16] \\
$K$ & Steady state parameter & 167.7 & $\mu \mathrm{gL}^{-1}$ & Simulations \\
\hline
\end{tabular}

The solution to IVP (2.1) is,

$$
C(t)=C_{\infty}^{+} \frac{1-\frac{C_{\infty}^{-}}{C_{\infty}^{+}}\left(\frac{C_{0}-C_{\infty}^{+}}{C_{0}-C_{\infty}^{-}}\right) e^{-\sqrt{(\mu-F)^{2}+4 C_{i} F \frac{\mu-F}{K}} t}}{1-\left(\frac{C_{0}-C_{\infty}^{+}}{C_{0}-C_{\infty}^{-}}\right) e^{-\sqrt{(\mu-F)^{2}+4 C_{i} F \frac{\mu-F}{K}} t}},
$$

where, $C_{\infty}^{-}$and $C_{\infty}^{+}$are given by,

$$
C_{\infty}^{ \pm}=\frac{K}{2}\left(1 \pm \sqrt{1+4 \frac{C_{i} F}{\mu-F}}\right) .
$$

The steady-state solution is $\lim _{t \rightarrow \infty} C(t)=C_{\infty}^{+}$. For this paper, we assume $\mu>F$, which is reasonable for our data set because $\mu>2 F$. If $\mu<F$, then physically this scenario corresponds to flushing the chlorophyll from the lake. This case is mathematically interesting but outside the regime of applicability for the physical case so we neglect it when running simulations. 
FORECASTING ALGAL BLOOM LAGS AND STABILITY

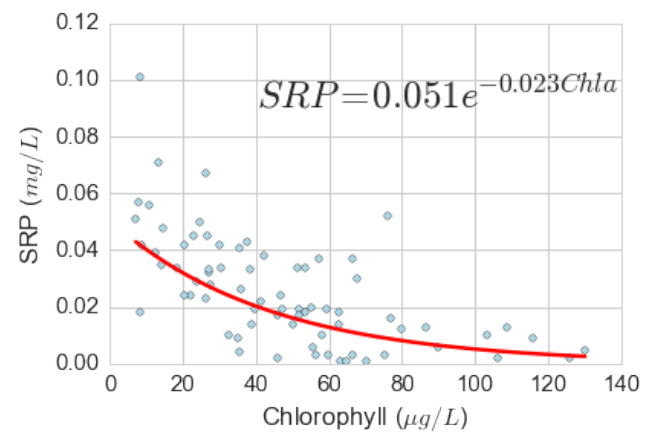

FIG. 4. 2015 REU data to examine the relationship between CHL-a and SRP to get an estimate for chlorophyll saturation level.

The flushing rate and growth rate were estimated from DNR data and similar values were also confirmed in [13]. To estimate $C_{\infty}^{+}$, previous field and laboratory observations obtained by an REU and the DNR were analyzed to get an estimate $[4,17]$. Figure 4 shows a plot of chlorophyll concentration against soluble reactive phosphorus. This data, collected by the REU, was analyzed to get an estimation of the saturation point under nutrient limited conditions, then this estimate was checked against previous years of DNR data. An estimate of $140 \mu \mathrm{g} / \mathrm{L}$ of chlorophyll was observed from the REU data and a maximum value of $200 \mu \mathrm{g} / \mathrm{L}$ was found in the DNR data. To reconcile the difference, an estimate of $175 \mu \mathrm{g} / \mathrm{L}$ was used. The results are robust to changes in the steady state value. For a given $\mu$, it takes approximately 3 weeks to reach the steady state for any saturation level between 130 to $250 \mu \mathrm{g} / \mathrm{L}$.

A carrying capacity was estimated using the previous value for $C_{\infty}^{+}$in equation (2.2) to get a value of $175 \mu \mathrm{g} / \mathrm{L}$. The standard deviation was determined by looking at DNR data [16] to get a value of $29 \mu \mathrm{g} / \mathrm{L}$. To run the first set of simulations, all parameters were held fixed except for the saturation value which was assumed to be normally distributed around mean $175 \mu \mathrm{g} / \mathrm{L}$ with standard deviation $29 \mu \mathrm{g} / \mathrm{L}$. In addition, a flushing rate of $.13 \mathrm{~d}^{-1}$ and a growth rate of $.3 \mathrm{~d}^{-1}$ were used, as obtained from DNR data [16]. The simulation was run using 100 realizations over a simulation time of 4 weeks. The realizations are indicated by the black lines and the average growth profile was computed and is indicated by the white dashed curve in Figure 5. IVP (2.1) was solved numerically using lsoda from the FORTRAN library odepack embedded within Scipy [12].

This model assumes an initial condition immediately after a large flushing event when the chlorophyll concentration is low, set to $10 \mu \mathrm{g} / \mathrm{L}$, and predicts what will happen if there is not another large rain event to flush the chlorophyll out of the lake. Under these conditions, the model shows that with average growth the population will hit a saturation level in approximately 3 weeks as shown in Figure 5. This result corresponds well with what is found in the DNR data that after a flushing event the chlorophyll levels take about 3 weeks to hit saturation levels because the doubling time for M. aeruginosa is approximately 3-5 days and there is a lag time for them to gather nutrients before starting to rapidly grow. This model confirms the fact that there is a lag time for algae growth after a flushing event.

A second set of simulations were also run with varying growth rate, $\mu=0.3 \pm$ $0.1 \mathrm{~d}^{-1}$. The other parameters are held fixed with a flushing rate a flushing rate of .13 $\mathrm{d}^{-1}$ and a saturation level of $175 \mu \mathrm{g} / \mathrm{L}$. The results are shown in Figure 6. This figure 


\section{JONES, A. FRIDERES, AND B. KRAMER}

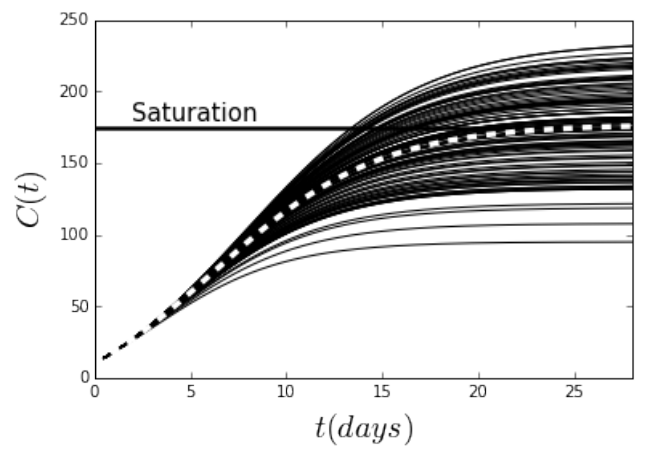

FIG. 5. Envelope of solutions after 100 realizations with varying steady-state solution $C_{\infty}^{+}=$ $175 \pm 29 \mu \mathrm{g} / L$. The horizontal line indicates chlorophyll saturation and the thick dotted line indicates average concentration growth.

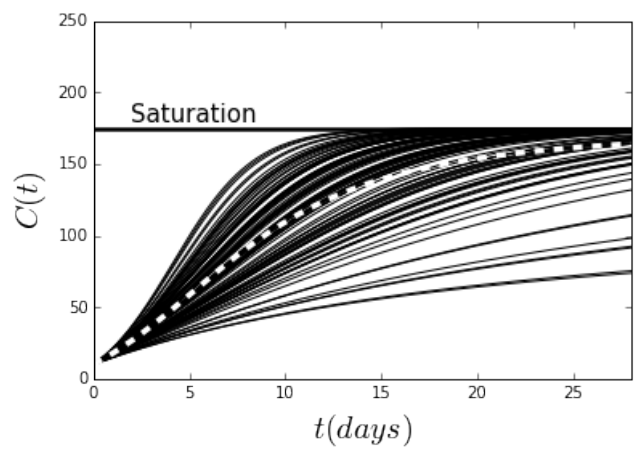

FIG. 6. Envelope of solutions after 100 realizations with varying growth parameter, $\mu=0.3 \pm$ $0.1 d^{-1}$. The horizontal line indicates chlorophyll saturation and the thick dotted line indicates average concentration growth.

also shows that the population takes approximately 3 weeks to hit saturation level. However, in Figure 5 solutions could end up less than or greater than the saturation level, while in Figure 6 all solutions are bounded by the saturation level.

To account for variation in the data and to test the sensitivity of the results, additional simulations are run with other growth parameter values. Figure 7 shows simulations being run with the growth parameter between 0.2 to $0.7 \mathrm{~d}^{-1}$ with the same standard deviation of $0.1 \mathrm{~d}^{-1}$. With higher growth rates the sample growth profiles converge more quickly to the average growth profile. In addition, as $\mu$ increases the saturation level is reached more quickly. It takes the average growth profile about 20 to 25 days to reach saturation when $\mu=0.3$ and it takes about 10 days when $\mu=$ 0.8 . This is intuitive because when algae grow at a faster rate they should reach the saturation level more quickly.

\section{Bloom Stability.}

3.1. Bloom Model. Simulating cyanobacteria growth was done by using a parabolic partial differential equation modified from Johnk's phytoplankton competition model [6]. Cyanobacteria are only considered in this model because little competition exists from other phytoplankton species that influence cyanobacterial growth; 
FORECASTING ALGAL BLOOM LAGS AND STABILITY
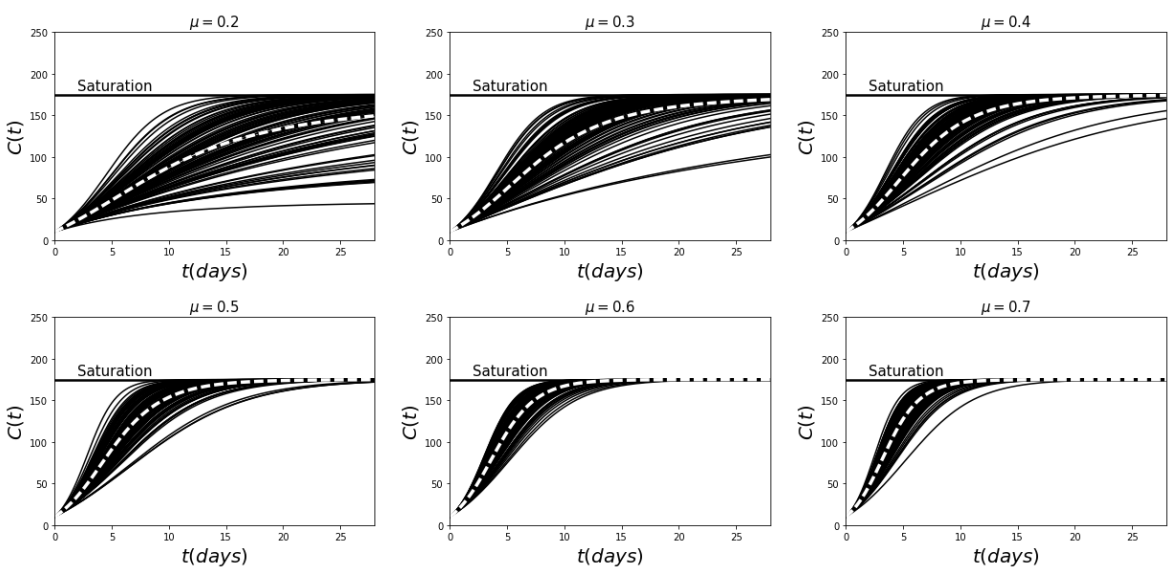

FIG. 7. Envelope of solutions after 100 realizations with varying growth parameters. The horizontal line indicates chlorophyll saturation and the thick dotted line indicates average concentration growth.

these other species are also not bothersome or toxic to Tainter Lake. It should be noted that this model is not used to preduct cyanobacteria concentration at a certain time but it will be used to predict whether the given lake conditions are viable for an algae bloom to occur. This model is seperate from model (2.1) and provides different solutions to solve the problem.

The chlorophyll concentration is modeled here since there was only available data on amount of chlorophyll in the lake. Cyanobacteria population can be backed out of the model since there is a strong positively linear relationship between chlorophyll and population. Due to this relationship, linear regression equations have been modeled so once the chlorophyll level is known the population can be estimated from the regression equation.

Chlorophyll is a byproduct of algal growth which is influenced by phosphorus, light availability, temperature, and turbulence. The chlorophyll concentration dynamic is described by:

$$
\left\{\begin{array}{l}
\frac{\partial C}{\partial t}=\mu(I, T) C-m(T) C+\frac{\partial}{\partial z}(v(T) C)+\frac{\partial}{\partial z}\left(D_{z} \frac{\partial C}{\partial z}\right) \\
l v_{\text {surface }} C(0, t)-D_{z} \frac{\partial C}{\partial z}=0 \\
C(1, t)=0 \\
C(z, 0)=f(z)
\end{array}\right.
$$

Where $C(z, t)$ denotes the chlorophyll concentration $(\mu \mathrm{g} / \mathrm{L})$ at time $t(\mathrm{~s})$ and depth $z(\mathrm{~m})$ with $l$ being the bottom depth, $I(z, t)\left(\mu \mathrm{mol} / \mathrm{m}^{2} \mathrm{~s}\right)$ denotes the intensity of the light that is available for photosynthesis and $T(z, t)$ denotes the temperature in Celsius. Temperature was gathered from previous DNR data and irradiance will be calculated as it depends on the amount of chlorophyll in the water, since more chlorophyll indicates murkier waters. Further, $\mu(I, T)$ represents the specific growth rate and is dependent upon the amount of light, temperature and heat that the cyanobacteria receives; conversely, $m(T)$ represents the mortality rate which is assumed to be solely dependent on temperature. The term $v(T)(\mathrm{m} / \mathrm{s})$ is the vertical velocity and since cyanobacteria have gas vacuoles that make them float their vertical float 
velocity and the chlorophyll that floats with them will be positive. The magnitude of the velocity is dependent upon the dynamic viscosity of water which is dependent on temperature. Lastly, $D_{z}$ is the vertical turbulent diffusivity which can be obtained from DNR data on the momentum and temperature of the water. A zero-mass flux continuity boundary condition is imposed at the surface of the lake since chlorophyll cannot leave or enter the lake and the concentration of chlorophyll stays continuous throughout the depth of the lake. A zero concentration boundary condition is imposed at the bottom of the lake. We assume no chlorophyll exists at the bottom of the lake which is reasonable becuase light does not reach the bottom of the lake for cyanobacteria to thrive and produce chlorophyll. Several initial conditions were used and did not have a large impact on the results.

The underwater light intensity may change due to increased chlorophyll because of a larger number of cyanobacteria. This relationship can be described with a LambertBeer's law:

$$
I(z, t)=I_{\text {in }}(t)(1-r) \exp \left(-\int_{0}^{z} \kappa C(\sigma, t) d \sigma-K_{b g} z\right) .
$$

Where $I_{i n}(t)$ is the incident light intensity that can be used for photosynthesis at the surface. Wave reflectivity is represented as $r$, implying $(1-r)$ corrects for any reflection losses at the surface. The light attenuation coefficient for cyanobacteria, $\kappa$, represents how easily light can be penetrated due to the cyanobacteria biomass. The background attenuation coefficient, $K_{b g}$, represents how easily the water can be penetrated by light due to non-phytoplankton, and $\sigma$ is an dummy integration variable accounting for the non-constant cyanobacteria distribution in depth. To find the initial irradiance $I_{i n}(t)$, the following relation is used:

$$
I_{\text {in }}=\left(1-0.65 C_{l}^{2}\right) I_{\text {sol }},
$$

where $C_{l}$ represents the amount of cloud cover $\left(0<C_{l}<1\right)$ and $I_{\text {sol }}$ is the amount of solar radiation for a sky without cloud cover.

The growth rate due to irradiance is described by a Monod equation, which relates microorganism growth rate to the concentration of a limiting nutrient in the environment. Irradiance is focused on as the limiting nutrient because Tainter Lake is highly eutrophic and the cyanobacteria can get all of the phosphorus that they need to grow. However, their phosphorus uptake rate does have some impact on their growth rate which then impacts the lake's bloom rate, which will be taken into account in future work. The relationship between growth rate and light intensity is described using a Monod model:

$$
\mu(I, T)=\frac{\mu_{\max }(T) I}{\mu_{\max }(T) / \alpha+I},
$$

where $\mu_{\max }(T)$ is the maximum growth rate at the light-saturation point as a function of temperature, which is where increases in light intensity do not increase the photosynthetic rate; and $\alpha$ is the initial slope of the growth curve under lightlimited conditions. The maximum growth rate is determined from the dark reaction of photosynthesis, which does not need light to occur, and because of the enzymes used it is temperature dependent. The slope $\alpha$ is determined from the light reaction of photosynthesis which is dependent on light intensity and absorption but not on temperature.

The maximum growth rate is modeled by an Arrhenius relationship which describes the temperature dependence on growth rates. The growth rate increases with 
increases in temperature until an optimum temperature, $T_{\text {opt }}$, is reached and then the growth rate decreases. The maximum growth rate is modeled as:

$$
\mu_{\max }(T)=\mu_{\max }\left(T_{o p t}\right)\left[1+b\left(\left(R_{1}^{T-T_{o p t}}-1\right)-\frac{\ln \left(R_{1}\right)}{\ln \left(R_{2}\right)}\left(R_{2}^{T-T_{o p t}}-1\right)\right)\right],
$$

where $\mu_{\max }\left(T_{\text {opt }}\right)$ is the maximum growth rate at the optimum temperature, and $R_{1}, R_{2}$, and $b$ describe the shape of the optimum curve. The mortality rate is dependent on temperature and is assumed to grow exponentially. This assumption is made since growth rate increases with temperature however when there are more bacteria there is more competition for nutrients and the environment cannot sustain as many new cyanobacteria so, more must die in order to keep balance. The mortality rate follows a $Q_{10}$ relationship, which describes the temperature sensitivity of the mortality rate due to increases in temperature by $10^{\circ} \mathrm{C}$. The relationship looks like:

$$
m(T)=m(20) Q^{\frac{T-20}{10}},
$$

where $m(20)$ is the mortality rate at $20^{\circ} \mathrm{C}$ and $Q$ is the factor by which the mortality rate increases. A reference temperature of $20^{\circ} \mathrm{C}$ is used since that is a typical water temperature.

The vertical velocity is a function of dynamic viscosity since the higher the viscosity, the thicker the water, the harder it is for cyanobacteria to float and thus the slower they float to the surface. Further, dynamic viscosity varies with temperature, the lower the temperature the higher the viscosity. The relationship between vertical velocity and temperature is modeled as:

$$
v(T)=\frac{\eta(20)}{\eta(T)} v(20)
$$

where $\eta(T)$ is the dynamic viscosity dependent on temperature, and $\eta(20)$ and $v(20)$ are the dynamic viscosity and vertical velocity at the reference temperature of $20^{\circ} \mathrm{C}$. Dynamic viscosity is dependent on temperature and this relationship is modeled as $[3]$ :

$$
\eta(T)=\frac{1.78 \times 10^{-3}}{1+0.0337 T+0.00022 T^{2}} .
$$

The term, $\mu(I, T) C-m(T) C$ describes the net growth or mortality of cyanobacteria in the lake and contributes to whether the population is growing or shrinking. If $\mu>m$, it is a source term and there is a gain in concentration; if $\mu<m$, it is a sink term and there is a loss in concentration. The term $\frac{\partial}{\partial z}(v(T) C)$ describes the advection of the population and contributes to how the population is concentrated at different depths. Since cyanobacteria have a positive floating velocity this means they will advect to the surface of the lake with growth, and the density at a given depth can change due to changes in vertical velocity at that depth. The term $\frac{\partial}{\partial z}\left(D_{z} \frac{\partial C}{\partial z}\right)$ describes the diffusion of the population and contributes to the population evenly spreading over the depth, which means areas with low density gain more while areas with particularly high density lose some of that density.

3.2. Model Analysis. IBVP (3.1) was analyzed by nondimensionalizing the model with, $\widetilde{t}=\frac{D_{z}}{L^{2}} t$ and $\widetilde{z}=\frac{z}{L}$ where $L$ is the depth of the euphotic layer. Introducing this scaling into equation (3.1) yields, 


$$
\left\{\begin{array}{l}
\frac{\partial C}{\partial \widetilde{t}}=\frac{\partial^{2} C}{\partial \widetilde{z}^{2}}+P_{e} \frac{\partial C}{\partial \widetilde{z}}+G C \\
P_{e} C(0, \widetilde{t})-\frac{\partial C(0, \widetilde{t})}{\partial \widetilde{z}}=0 \\
C(1, \widetilde{t})=0 \\
C(\widetilde{z}, 0)=f(\widetilde{z}) .
\end{array}\right.
$$

where the Peclet number, $P_{e}=\frac{v L}{D_{z}}$, is the ratio of the advection rate to the diffusion rate, the growth Peclet number, $G=\frac{\mu L^{2}}{D_{z}}$, is the ratio of the growth rate to the diffusion rate. An inequality is derived in Section 3.3 that compares $P_{e}$ to $G$ to indicate whether conditions are conducive for a bloom to occur.

\begin{tabular}{cllll}
\hline Parameter & Description & Value & Units & Source \\
\hline Bloom Stability & & & \\
$v$ & Sinking or flotation velocity & $1.4 \mathrm{e}^{-4}$ & $\mathrm{~m} \mathrm{~s}^{-1}$ & Jöhnk [6] \\
$L$ & Euphotic layer length & 2.81 & $\mathrm{~m}$ & DNR [16] \\
$P_{e}$ & Peclet number & $0-4$ & - & Simulations \\
$G$ & Growth Peclet number & $0-12$ & - & Simulations \\
$\lambda$ & Degree of freedom & $\pi / 2-\pi$ & - & Simulations \\
\hline
\end{tabular}

The following two subsections report the conclusions from investigating the IBVP (3.2) both analytically and numerically. An analytic solution is used to determine a stability condition signifying when the concentration "blows-up" versus when the concentration collapses. This condition is independent of the initial condition and depends solely on $P_{e}, G$, and an eigenvalue that arises from solving the IBVP (3.2). A numerical solution is used for efficient experimentation over a large parameter space and, most importantly, for a variety of initial conditions. Note that an analytic solution is expressed as an infinite Fourier series with coefficients that are expressed as integrals involving the initial condition. The series needs to be truncated and even a rather innocuous initial condition could lead to Fourier coefficients with no closedform integral. In summary, a numerical solution is inevitable for experimentation so a numerical scheme like the one outlined below is the most efficient means for such exploration and the value of the analytic solution is the stability condition for the concentration.

3.3. Bloom Stability Condition. IBVP (3.2) was solved as follows:

1. The transform $C=e^{\left(-\left(\frac{P_{e}}{2}\right)^{2}+G\right) \widetilde{t}-\frac{P_{e}}{2} \widetilde{z}} u$ was used to reduce equation (3.2) to a simple diffusion IBVP in terms of $u$.

2. Separation of variables was used construct a Fourier series expansion for $u$ after the boundary conditions were imposed.

3. The initial condition was left in terms of $f(\widetilde{z})$ for generality.

4. The solution, $u$, was substituted into the equation for $C$ in step (1) above.

These steps yield the concentration profile,

$$
C(\widetilde{z}, \widetilde{t})=\sum_{n=0}^{\infty} c_{n} e^{\left(-\lambda_{n}^{2}-\left(\frac{P_{e}}{2}\right)^{2}+G\right) \widetilde{t}}\left(\sin \left(\lambda_{n} \widetilde{z}\right)+\frac{2}{3 P_{e}} \lambda_{n} \cos \left(\lambda_{n} \widetilde{z}\right)\right),
$$

where the Fourier coefficients $c_{n}$ are computed using the integral:

$$
c_{n}=\frac{1}{2} \int_{0}^{1} f(\widetilde{z}) e^{\left(\frac{P_{e}}{2}-i \lambda_{n}\right)} \widetilde{z} d \widetilde{z} .
$$




\section{FORECASTING ALGAL BLOOM LAGS AND STABILITY}

The $\lambda_{n}$ are computed by solving the nonlinear eigenvalue problem,

$$
\sin \left(\lambda_{n}\right)+\frac{2}{3 P_{e}} \lambda_{n} \cos \left(\lambda_{n}\right)=0,
$$

for a given Peclet number, $P_{e}$.

All expressions dependent upon $\widetilde{z}$ are bounded and if the initial condition is continuous, $f(\widetilde{z}) \in C([0,1])$, as is often the case, then all $c_{n}$ converge and are bounded as well. The terms dependent upon $\widetilde{t}$ are the main focus of this analysis since they have the potential to increase to infinity, which would make the series diverge and indicate a bloom has occurred. If the eigenvalues, $\lambda_{n}$, satisfy the condition,

then the concentration decays exponentially and the series converges to zero implying cyanobacteria population collapse, that is, no bloom occurs. Otherwise the concentration tends to infinity as $t$ tends to infinity and the solution diverges implying the cyanobacteria population grows without bound, that is, a bloom occurs. Of course the cyanobacteria population in this case becomes nutrient limited for the lake considered but this case is investigated using a different model in Section 2.

The eigenvalues, $\lambda_{n}$, play a prominent role in the stability analysis of equation (3.3) so it is important to investigate their behavior. One can use Newton's method to solve for $\lambda_{n}$ and the formulation given in equation (3.4) is most stable. However, it is simpler to study the features of $\lambda_{n}$ if equation (3.4) is rewritten as,

$$
\tan \left(\lambda_{n}\right)=-\frac{2}{3 P_{e}} \lambda_{n}
$$

The nonlinear equation (3.6) reveals that the eigenvalues, $\lambda_{n}$, have the following features for all $n \geq 0$, see Figure 8:

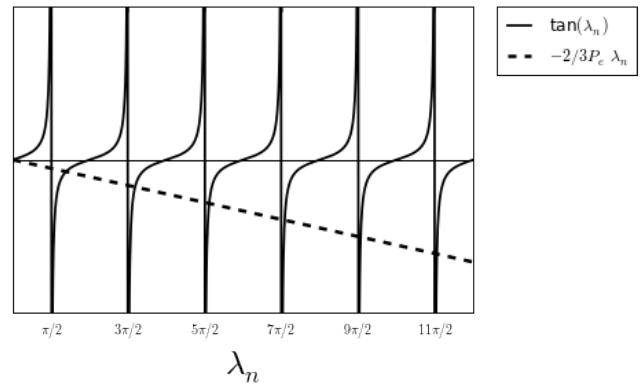

FIG. 8. For varying values of $\lambda_{n}$, the solid lines represent $\tan \left(\lambda_{n}\right)$ and the dotted line represents $-\frac{2}{3 P_{e}} \lambda_{n}$. Their intersection shows where the eigenvalue condition (3.6) is satisfied.

- monotonically increasing, $\lambda_{0}<\lambda_{1}<\lambda_{2}<\ldots$

- if $P_{e} \rightarrow \infty$, then $\lambda_{n} \rightarrow n \pi$

- if $P_{e} \rightarrow 0$, then $\lambda_{n} \rightarrow \frac{(2 n-1) \pi}{2}$

- $\frac{(2 n-1) \pi}{2}<\lambda_{n}<n \pi$ 
- $\lambda_{n} \sim \frac{2 n-1}{2} \pi+\frac{3 P_{e}}{\pi(2 n-1)}$ asymptotically with $n>P_{e}$

Note that $\lambda_{0}=0$ yields the trivial solution for the concentration so this case is ignored. These features reveal that if the stability condition equation (3.5) fails for $\lambda_{1}$, it will fail for all remaining eigenvalues. Newton's method was run on equation (3.4) for each $P_{e}$ with a tolerance of $10^{-5}$ in order to determine $\lambda_{1}$. Figure 9 shows the range of values and the asymptotic behavior of $\lambda_{1}$, notice that indeed $\frac{\pi}{2}<\lambda_{1}<\pi$.

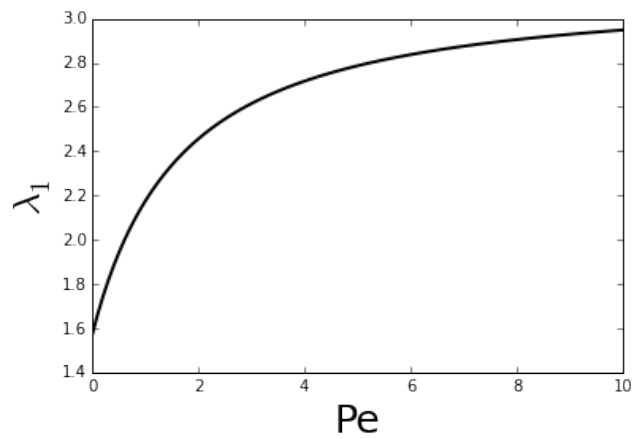

FIG. 9. Given a value for $P_{e}, \lambda_{1}$ is determined by using Newton's method and equation (3.4).

Now that the role of $\lambda_{1}$ has been established in inequality (3.5) and it is clear that Newton's method can be used to determine its values, a stability condition can be written in terms of an upper bound on the growth parameter $G$. The stability condition for the non-dimensional case is,

$$
G<\lambda_{1}^{2}+\frac{P_{e}^{2}}{4}
$$

This condition reveals the circumstances under which the concentration remains bounded and consequently leads to a population collapse. So inequality (3.7) will hence be referred to as the "bloom stability condition." When the bloom stability condition (3.7) is satisfied the population collapses and the concentration tends to zero otherwise the bloom grows unconditionally and is classified as unstable. The bloom stability condition is best summarized using the plot in Figure 10. Notice that it divides the first quadrant into two regions, one for population collapse and one for population growth.

If the cyanobacteria are in a nutrient-limited environment, then their growth depends on phosphorus uptake. Under these circumstances concentration collapse or growth hinges on the magnitude of the turbulent diffusivity, $D_{z}$. Rewriting inequality (3.7) in dimensional terms yields an upper bound on the turbulent diffusivity, $D_{z}$,

$$
0<\left(\frac{\lambda_{1}}{L}\right)^{2} D_{z}^{2}-\mu D_{z}+\left(\frac{v}{2}\right)^{2}
$$

If the advective velocity, $v$, and the euphotic layer, $L$, are held fixed, then Figure 11 shows that that there is a condition under which the concentration will grow or collapse. Expressed in dimensional terms, the condition under which the population will collapse is now, 


\section{FORECASTING ALGAL BLOOM LAGS AND STABILITY}

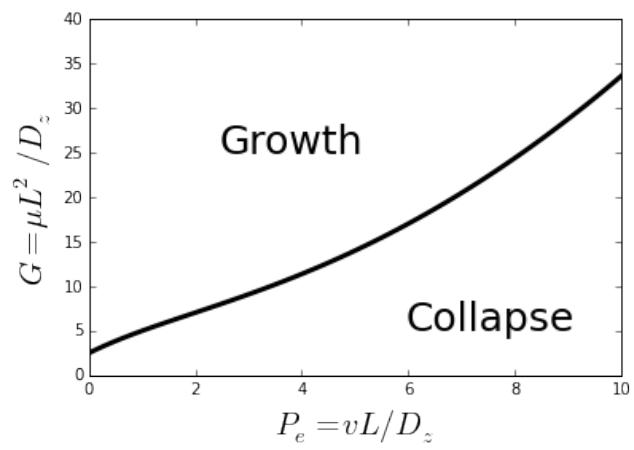

FIG. 10. The bloom stability condition separates the quadrant into two regions, if $G<\lambda_{1}^{2}+\frac{P_{e}^{2}}{4}$, then the population experiences collapse, otherwise the population experiences unbounded growth.

$$
D_{z}>\frac{\mu}{2}\left(\frac{L}{\lambda_{1}}\right)^{2}\left[1+\sqrt{1-\left(\frac{\lambda_{1} v}{\mu L}\right)^{2}}\right]
$$

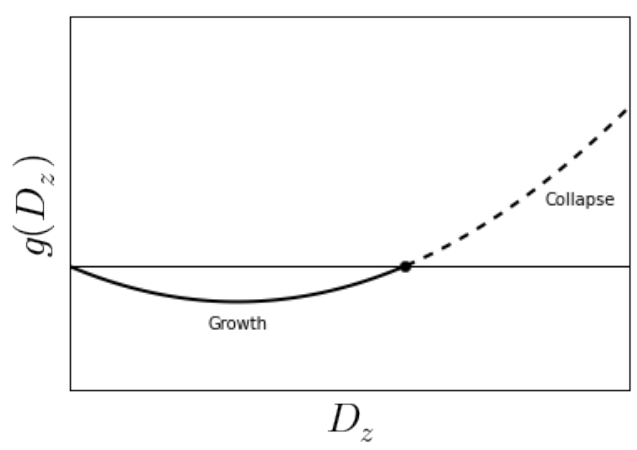

FIG. 11. The bloom stability condition in dimensional terms. In a nutrient-limited environment, the bound on the turbulent diffusivity, $D_{z}$, establishes the limitation on growth.

Given that $\frac{\pi}{2}<\lambda_{1}<\pi$ and suppose that $\left(\frac{\lambda_{1} v}{\mu L}\right)^{2} \ll 1$, then inequality (3.8) simplifies to a similar criterion provided in [19],

$$
D_{z}>\frac{4 \mu L^{2}}{\pi^{2}} .
$$

Moreover, inequality (3.8), establishes a minimum criteria for the growth parameter, $\mu$. If the expression under the radical is to remain real, then

$$
\mu>\frac{\lambda_{1} v}{L},
$$

to maintain concentration growth. This requirement implies a nutrient limitation and the existence of a carrying-capacity for the cyanobacteria population. A modified 
model based on the one given in [13] is given in Section 2 where a carrying-capacity is included to forecast the concentration levels in the lake in a nutrient-limited environment. Again, since $\frac{\pi}{2}<\lambda_{1}<\pi$, inequality (3.10) is bounded below and can be written,

$$
\mu>\frac{\pi v}{2 L} .
$$

Combining inequalities (3.9) and (3.11) yields a simpler stability criterion in a nutrient-limited environment where the growth parameter is suitably large enough to support growth. Again, if $\left(\frac{\lambda_{1} v}{\mu L}\right)^{2} \ll 1$ then the inequality is completely dependent upon the sinking velocity and the euphotic layer,

$$
D_{z}>\frac{2}{\pi} v L
$$

If the turbulent diffusivity satisfies this criterion, then the population collapses, otherwise it grows. Inequality (3.12) may be used in the event that one assumes the growth parameter is high enough to support growth and is searching for a turbulent diffusivity that can bring about population collapse in this "worst-case scenario." Otherwise, one can look to bring about collapse by limiting $\mu$ and increasing $D_{z}$.

Currently there is not data available to verify this model. However there are plans for a real time sensor that can collect chlorophyll-a concentration and turbulence of the water. Once this data is collected this model can be verified.

4. Conclusion. A logistic model (2.1) was proposed to forecast the chlorophyll-a concentration in Tainter Lake. This model was shown to have a steady-state solution (2.2), that is a function of the growth rate, thus indicating that the $M$. aeruginosa population is time and resource dependent. After a flushing event occurs there is lag before the population grows and the chlorophyll-a increases to the steady-state. One can use the model to test a variety of parameters for the growth rate to forecast concentration levels over time.

A second model (3.1) was proposed for analysis to better understand both the biologic and limnogic conditions that cause algae blooms. This model was used to arrive at a bloom-stability condition solely dependent upon the sinking velocity and the euphotic layer, (3.12). One can use the model to test if a combination parameters will bring about population collapse.

These models may be used in tandem to make predictions involving proposed solutions to mitigate the algal blooms in Lake Tainter. Both models suggest lowering the growth rate of algae can prevent the population from growing too large. The growth rate can be lowered by limiting the amount of nutrients that are allowed into the lake. Model (3.1) suggests increasing turbulence can bring about population collapse. This can be done by undamming the lake or by installing lake bubblers that manually aerate and disturb the lake.

Acknowledgments. The authors would like to acknowledge the assistance of Stephen Nold and William James for their invaluable guidance in the writing of this manuscript.

REFERENCES 


\section{FORECASTING ALGAL BLOOM LAGS AND STABILITY}

[1] M. Boyle AND K. Kiel, A survey of house price hedonic studies of the impact of environmental externaities, Journal of Real Estate Literature, 9 (2001), pp. 117-144, http://aresjournals. org/doi/abs/10.5555/reli.9.2.23u082061q53qpm3?code=ares-site (accessed 2018/01/10).

[2] W. DodDs, Freshwater Ecology: Concepts and Environmental Applications, Academic Press, San Diego, C.A., 2002.

[3] K. Hutter And K. Johnk, Continuum Methods of Physical Modeling - Continuum Mechanics, Dimensional Analysis, Turbulence, Springer, Berlin, G.R., 2004.

[4] W. James, S. Nold, L. Anderson, , R. Fleck, H. Lieffort, N. Loeven, L. Provos, M. VANDENBERG, AND A. Wilson, Limnological conditions in tainter and menomin reservoirs: Interim report 2017. Discovery Center - Sustainability Research and Rehabilitation, Menomonie, WI 54751, 2017.

[5] A. JansSEn AND S. T. ET AL, Alternative stable states in large shallow lakes?, Journal of Great Lakes Research, 40 (2014), pp. 813-826, https://doi.org/10.1016/j.jglr.2014.09.019.

[6] K. Johnk And J. H. ET AL, Summer heatwaves promote blooms of harmful cyanobacteria, Global Change Biology, 14 (2008), pp. 495-512, https://doi.org/10.1111/j.1365-2486.2007. 01510.x.

[7] J. Kronkamp, A. V. D. Heuvel, and L. Mur, Phosphorus uptake and photosynthesis by phosphate-limited cultures of the cyanbacterium microcystis aeruginosa, British Phycological Society, 24 (1989), pp. 347-355, https://doi.org/10.1080/00071618900650361.

[8] C. Krysel, E. M. Boyer, C. Parson, And P.Welle, Lakeshore property values and water quality: Evidence from property sales in the mississippi headwaters region. Legislative Commission on Minnesota Resources by the Mississippi Headwaters Board and Bemidji State University, 2003.

[9] H. Paerl, R. Fulton, P. Moisander, and J. Dyble, Harmful freshwater algal blooms with an emphasis on cyanobacteria, The Scientific World, 1 (2001), pp. 76-113, https://doi. org/10.1100/tsw.2001.16.

[10] H. Paerl and T. Otten, Harmful cyanbacterial blooms: Cause, consequences, and controls, Microbial Ecology, 65 (2013), pp. 995-1010, https://doi.org/10.1007/s00248-012-0159-y.

[11] H. Pedrotti, C. Delaney, E. Melly, and D. Ferguson, Economic impacts of algae: An hedonic pricing analysis of water quality valuation. Working paper, 2017.

[12] SCIPY.ong, scipy.integrate.odeint, 2004, http://docs.scipy.org/doc/scipy/reference/generated/ scipy.integrate.odeint.html (accessed 2016/07/29).

[13] D. Soballe AND S. Threlkeld, Advection phytoplankton biomass and nutrient transformations in a rapidly flushed impoundment, Archiv fur Hydrobiologie, 105 (1985), pp. 187-203.

[14] United States Geographical Survey, Usgs 05367500 red cedar river near colfax, wi, 2015, http://nwis.waterdata.usgs.gov/usa/nwis/uv/?cb_00060=on\&cb_00065=on\&format= html\&site_no=05367500\&period $=$ \&begin_date $=2015-05-01 \&$ end_date $=2015-10-10 \quad$ (accessed 2016/07/29).

[15] WeAther UndERGROUND, Weather history for colfax, wi, 2015, https://www.wunderground. $\mathrm{com} /$ personal-weather-station/dashboard?ID=KWICOLFA3\&scrollTo=historyTable\# history/s20150501/e20150501/mdailyl (accessed 2016/07/29).

[16] Wisconsin Department of Natural Resources, Phosphorus Total Maximum Daily Loads, 2012.

[17] Wisconsin Department of Natural Resources, Lake water quality 2015 annual report: Tainter lake middle basin, 2015, https://dnrx.wisconsin.gov/swims/public/ reporting.do?type $=10 \&$ action $=$ post \&stationNo $=173215 \&$ year $1=2015 \&$ format $=$ html $\quad($ accessed 2016/07/29).

[18] Wisconsin Department of Natural Resources, Tainter lake, 2015, http://dnr.wi.gov/ lakes/lakepages $/$ LakeDetail.aspx wbic $=2068000 \&$ page $=$ facts $($ accessed 2016/07/29).

[19] K. Wong, J. LeE, And I. Hodgkiss, A simple model for forecast of coastal algal blooms, Estuarine, Coastal, and Shelf Science, 74 (2007), pp. 175-196, https://doi.org/10.1016/j. ecss.2007.04.012. 